\title{
Re: Comparative Effectiveness of Combined Low- and Standart-Dose Trospium and Solifenacin for Moderate Overactive Bladder Symptoms in Elderly Men and Women
}

\author{
Kirill V. Kosilov, Sergey A. Loparev, Marina A. Ivanovskaya, Liliya V. Kosilova
}

Urol Int 2014;93:470-473.

\section{EDITORIAL COMMENT}

Overactive bladder $(\mathrm{OAB})$ treatment is also a problem in elderly people. Treatment alternatives are limited especially in elderly patients who were resistant to standart antimuscarinic treatments. In this study, the authors examined a group of elderly patients with OAB, who had an episode of incontinence (EI) three or less a day and unsatisfied with the treatment, and low dose (Trospium 15mg/day+Solifenacin $5 \mathrm{mg} /$ day) versus standart dose (Trospium 30mg/day+Solifenacin $10 \mathrm{mg} /$ day) trospium+solifenacin treatments were evaluated. The assignment of patients was random and blind in this placebo-controlled study. Urodynamic study, ICIQ-SF questionnaires and bladder diaries were used. Significant improvement in symptoms and urodynamic parameters were seen in both treatment groups. The frequency of El in both of the main groups decreased by almost 2-fold compared to the initial data. Side effects were more in standart dose group. They concluded that, as the treatment efficacy of both groups was similiar, combination of these drugs in standart doses for such patients is excessive. Also the authors discussed that synergistic effects of combination of antimuscarinics were more effective than high doses. Search for non-invasive tretment alternatives in antimuscarinic resistant $O A B$ is ongoing.

İlker Şen MD

\section{Re: Comparison of Partial Nephrectomy and Percutaneous Ablation for cT1 Renal Masses \\ Thompson RH, Atwell T, Schmit G, Lohse CM, Kurup AN, Weisbrod A, Psutka SP, Stewart SB, Callstrom MR, Cheville JC, Boorjian SA, Leibovich BC}

European Urology 2015;67(2):252-259.

\section{EDITORIAL COMMENT}

Partial nephrectomy (PN) and radical nephrectomy achieves similar oncological outcomes for cT1 renal tumors and European Association of Urology (EAU) guideline suggested PN for cT1 tumors whenever technically feasible. EAU guideline state that percutaneous ablation therapy should be limited for comorbid patients with small renal masses. Previous studies were controversy, percutaneous ablation therapy had similar or lower recurrence free survival rate when compared with PN. In this study the authors for the first time compared oncological outcomes of two different percutaneous ablation therapies including radiofrequency ablation (RFA) and cryoablation with PN for cT1 tumors. There were 1424 patients treated with PN $(n=1057)$, RFA ( $n=180)$ and cryoablation $(n=187)$ for T1a tumors and 379 patients treated with PN $(n=326)$ and cryoablation $(n=53)$ for T1b renal tumors. Local recurrence-free survival rate at 3 year was similar among three therapy (98\%) for T1a tumors. PN (99\%) and cryoablation (100\%) were significantly better metastases-free survival rates at 3 year compared with RFA (93\%) for cT1a tumors. For T1b tumors local recurrence free survival rates and distant metastases free survival rates were similar at 3 year for PN (96\%, 96\% respectively) and cryoablation (97\%, 92\% respectively). PN (93\%) had higher overall survival rates at 3 year for T1b patients compared with cryoablation $(74 \%)(p<0.001)$. Retrospective nature of study is a limitation. Significantly younger patients and lower Charlson scores among PN patients might have led to superior overall survival rates after PN and may be related with selection bias. This study encourage percutaneous ablation in patients with cT1 tumors who are unfit for surgery and may take part in guidelines in near future. 